Mauricio Leite de Oliveira' Joaquim Gonçalves Machado Neto $^{2}$

\title{
Segurança no Trabalho com Agrotóxícos em Citros: Aplicação com o Turbopulverizador e Preparo de Calda em Tanque de 2.000 L
}

\section{Work Safety in the Use of Pesticides in Citrus: Application with an Air- Assisted Sprayer and Preparation of Spray in a 2.600-L Tank}

'Engenheiro Agrônomo

'Professor Livre-Docente do

Departomento de Fitossanidade, FCAV/UNESP

Agradocimentos

Os autores agradecem dे

Fundação de Amparo à Pesquisa do

Estado de São Paulo-FAPESP pelo

auxilio concedido para a realização deste trabolho.
Os objetivos deste trabalho foram quantificar as exposições dérmicas e respiratórias proporcionadas pelas condições de trabalho na aplicação de agrotóxicos em cultura de citros com - turbopulverizador e no preparo de caldas em um tanque de $2.000 \mathrm{~L}$ de capacidade; avaliar a eficiência de medidas de proteção individual; determinar as regiões mais expostas do corpo dos trabalhadores e coletiva para esses trabalhadores; e classificar as condiçōes de trabalho, sem e com as medidas de proteção testadas, quanto à segurança ocupacional das recomendações de agrotóxicos registrados para o controle das principais pragas e doenças dessa cultura. Verificou-se, que para o tratorista e para o aplicador, pulverizando agrotóxicos em citros com o turbopulverizador, as medidas de proteção mais eficientes foram o conjunto AZR e a cabina Real. O conjunto AZR foi eficiente no controle da exposição do preparador de caldas, pois foi suficiente para tornar condições de trabalho inseguras em seguras. A região mais exposta do corpo, nas duas condições de trabalho estudadas, foram as mãos dos trabalhadores.

Palavras-chaves Agrotóxicos, Exposição Dérmica, Exposição Respiratória, Citrus.

The objectives of this study were: a) to quantify dermal and respiratory exposure resulting from work conditions in the application of pesticides on citrus crops using a air-assisted sprayer and by preparing the spray in a tank with a capacity of $2.000 \mathrm{~L} ; \mathrm{b}$ ) to evaluate the measures of individual protection for these workers; $c$ lo determine the areas of the worker's body most exposed; and d) to classify the work conditions with and without the protective measures tested, with regard to the occupational safery of the recommendations for the pesticides used in controlling the main pests and diseases of this crop. It was shown that for the tractor driver and sprayer operator applyng pesticides on citrus trees, the most efficient protective measures were the AZR ensemble and the Real cabin. The AZR ensemble was effective in controlling exposure of the spray preparer, because it was sufficient to turn work conditions from unsafe to safe. The area of the body most exposed under the two work conditions studied was the hands of the workers.

Keywords Pesticide, Dermal Exposure, Respiratory Exposure, Citrus. 


\section{Introdução}

A cultura de citros destaca-se com o quinto maior volume de agrotóxicos aplicados no Brasil, correspondendo a $4 \%$ do total das vendas de 2,5 bilhões de dólares no ano de 2000 (SINDAG, 2000). Por outro lado, os processos convencionais na aplicação desses agrotóxicos, nessa cultura, caracterizamse por alto desperdicio. Matuo (1988) constatou que a retenção no alvo foi de 30 a $45 \%$ dos 7 litros de calda aplicados por planta com o turbopulverizador. Nessas aplicações, a calda não retida nas plantas fica dispersa no ambiente, podendo atingir os trabalhadores envolvidos nessas atividades e causar-lhes intoxicações.

Na atividade de preparo de caldas, onde - operador trabalha com a formulação pura, ou seja, muito mais concentrada do que no momento da aplicação, também há possibilidade de exposição e risco de intoxicação ocupacional. Segundo Machado Neto (1997), no processo de tríplice lavagem, realizada imediatamente após o esvaziamento completo da embalagem, os trabalhadores no campo não tampam devidamente a embalagem e, com a agitação, ocorrem vazamento e contaminação externa da embalagem e das partes do corpo do trabalhador, principalmente das mãos.

As medidas de proteção que controlam as exposições ocupacionais aos agrotóxicos, atuam na prevenção do contato dos produtos com as vias de exposição do corpo. Em condições de aplicação de agrotóxicos no campo, $99 \%$, ou mais, da exposição total ocorre na via dérmica e apenas $1 \%$, ou menos, na via respiratória (WOLFE et al., 1972; VAN HEMMEN, 1992).

Portanto, o conhecimento das exposições dérmica e respiratória potenciais, das características tóxicas dos agrotóxicos e da eficiência das medidas de proteção é de grande importância para a adoção de estratégias de segurança mais efetivas, confortáveis, econômicas e aplicáveis, nas condições específicas de trabalho de campo com esses produtos.

De maneira geral, tem-se recomendado, como medida de proteção no trabalho com agrotóxicos, apenas o uso de equipamentos de proteção individual (EPIs). Entretanto, quando os trabalhadores têm acesso aos EPIs, eles são desconfortáveis e de uso insuportável em condições de campo, pois proporcionam grande retenção de umidade e de calor na superficie do corpo (BULL \& HATHAWAY, 1986).

O presente estudo objetivou quantificar as exposições dérmica e respiratória proporcionadas pelas condições de trabalho na aplicação de agrotóxicos em cultura de citros com o turbopulverizador e no preparo de caldas no tanque de $2.000 \mathrm{~L}$ de capacidade desse pulverizador; determinar as regiōes mais expostas do corpo dos trabalhadores; avaliar a eficiência de medidas de proteção individual e coletiva para esses trabalhadores; e classificar as condições de trabalho, sem e com as medidas de proteção testadas, quanto à segurança ocupacional das recomendações de agrotóxicos registrados para o controle das principais pragas e doenças dessa cultura.

\section{Material e métodos}

A segurança ocupacional das atividades na aplicação de agrotóxicos em cultura de citros com o furbopulverizador e no preparo de caldas em um tanque de $2.000 \mathrm{~L}$ foi calculada com os dados de exposição dérmica (ED) e de exposição respiratória (ER) proporcionadas aos trabalhadores em condições de campo. As avaliações das exposições do tratorista foram realizadas durante os anos de 1999 e 2000, em pomares localizados no município de Matão-SP. As avaliações do preparador de caldas foram realizadas na Faculdade de Ciências Agrárias e Veterinárias FCAV/UNESP, Câmpus de Jaboticabal, Jaboticabal, Estado de São Paulo, em tanque-padrão com capacidade de $2.000 \mathrm{~L}$ de calda.

Denominaram-se exposição dérmica potencial (EDP) e exposição respiratória potencial (ERP) as EDs e ERs avaliadas sem medidas de proteção e que, teoricamente, poderiam atingir as vias de exposição dérmica e respiratória dos trabalhadores. Denominaram-se exposição dérmica não controlada (EDNC) e exposição respiratória não controlada (ERNC) as exposiçōes não controladas pelas medidas de proteção.

As exposições do tratorista foram avaliadas em pulverizações de pomares de laranja da variedade Valência, com plantas de 4,5e 
$5,0 \mathrm{~m}$ de altura. $O$ turbopulverizador utilizado foi o modelo Arbus 2.000 Valência, marca Máquinas Agrícolas Jacto S.A., tracionado por trator Massey Ferguson, MF 275 , trabalhando em $3^{3}$ marcha reduzida e rotação de $1.650 \mathrm{rpm}$. O tanque desse pulverizador tem $2.000 \mathrm{~L}$ de capacidade, e sua turbina proporciona fluxo de ar de $19,5 \mathrm{~m}^{3}$ de ar/s, a $2.050 \mathrm{rpm}$. Os bicos utilizados nos ramais da barra de aplicação estavam com pontas J5-2 alternados com J5-3. O bico mais baixo estava a 1,2 $\mathrm{m}$ de altura do solo. O pulverizador foi calibrado para aplicar o volume de $10 \mathrm{~L}$ de calda por planta, com a pressão de 21 bar.

O tanque utilizado nas avaliações das exposições dérmica e respiratória do preparador de caldas foi o de um pulverizador da marca KO Máquinas Agrícolas LTDA, com capacidade de $2.000 \mathrm{~L}$, que possui altura de 1,50 $\mathrm{m}$ e boca de carga com diâmetro de $45 \mathrm{~cm}$. Este tanque foi adotado como padrão por ser utilizado tanto em pulverizadores de pistolas como em turbopulverizadores utilizados para aplicação de agrotóxicos na cultura de citros.

As embalagens utilizadas foram frascos de plástico polietileno tereftalato (PET) de $1 \mathrm{~L}$, com tampa plástica rígida de rosquear e "boca" com $4 \mathrm{~cm}$ de diâmetro. Cada repetição do abastecimento foi realizada com três embalagens, seguindo o seguinte procedimento: agitação vigorosa da embalagem tampada, retirada da tampa, colocação da suspensão de fungicida cúprico no tanque do pulverizador e realização da tríplice lavagem. A tríplice lavagem foi realizada colocandose $250 \mathrm{~mL}$ de água limpa na embalagem imediatamente após o esvaziamento, colocação da tampa e vigorosa agitação por 30 segundos, e deposição da calda no tanque do pulverizador. Esse procedimento foi repetido mais duas vezes.

As EDPs foram avaliadas em trabalhadores expostos às caldas contendo o cátion cobre do fungicida cúprico Cuprogarb 500 PM como traçador, conforme metodologia descrita e utilizada por Machado Neto (1997). Como vestimentas amostradoras das exposições dérmicas dos trabalhadores, foram utilizados macacões, tipo brim, de algodão, e luvas de algodão nas mãos. As exposições da face e dos pés foram avaliadas em absorventes higiênicos femininos afixados sobre uma máscara semifacial descartável e sobre o "peito" dos pés (PRADELA, 1998). As EDs foram avaliadas nas seguintes regiōes do corpo dos trabalhadores: cabeça + pescoço, face, mãos, braços, tórax-frente, tórax-atrás, coxas+pernas-frente, coxas+pernas-atrás $e$ pés.

As exposições foram avaliadas em períodos inferiores a uma hora de trabalho e extrapoladas para um dia de seis horas de exposição efetiva (MACHADO NETO, 1997).

As ERPs foram avaliadas em filtros de éster celulose, com porosidade de $0,8 \mathrm{~mm}$, utilizados em cassetes de bombas de fluxo continuo de ar, marca A. P. Buck, com vazão de $2 \mathrm{~L} / \mathrm{min}$. Os cassetes foram posicionados na região de respiração dos trabalhadores expostos às caldas contendo o cátion $\mathrm{Mn}^{+3}$ como traçador (OLIVEIRA, 2000). As ERPs foram avaliadas por períodos de aproximadamente três horas e extrapoladas para um dia de seis horas de exposição efetiva.

As EDPs e ERPs às caldas foram estimadas com os valores de $\mathrm{Cu}^{+2}$ e $\mathrm{Mn}^{+3}$ utilizados como traçadores e suas respectivas concentrações nas caldas aplicadas. Todas as atividades foram avaliadas com 10 repetiçōes.

Os macacões, seccionados em partes, imediatamente após as exposições, as luvas de algodão e os absorventes higiênicos femininos, utilizados como amostradores, e os filtros coletores das bombas pessoais foram imersos em solução de $\mathrm{HCl} \mathrm{0,2} \mathrm{N}$ para a solubilização dos cátions. Os amostradores permaneceram na solução solubilizadora por duas horas e, em seguida, foi filtrada uma aliquota em filtro de papel qualitativo nas amostras que continham o $\mathrm{Cu}^{+2}$ (MACHADO NETO \& MATUO, 1989). Nas amostras com $\mathrm{Mn}^{+3}$, procedeu-se apenas a retirada dos filtros de éster celulose das soluções. Os cátions foram quantificados nas aliquotas dessas soluções em espectrofotômetro de absorção atômica.

Para as avaliações das EDNCs, as vestimentas amostradoras foram utilizadas sob o equipamento de proteção individual (EPI) e quando da utilização dos equipamentos de proteção coletivos (cabinas e semicabina). As avaliações das EDNCs do tratorista foram realizadas em duas fases: na primeira, avaliaram-se o EPI e a Semicabina; e, na segunda, duas marcas de cabinas. 
O conjunto AZR, novo (sem sofrer nenhuma lavagem), foi avaliado para o tratorista $e$ para o preparador de caldas, como medida de proteção individual. Esse conjunto constituiu-se das seguintes vestimentas: blusa de mangas compridas, calças compridas e touca árabe com aba frontal, confeccionadas em tecido de algodão tratado com Teflon ${ }^{\circledR}$, para proporcionar hidrorrepelência, viseira de acetato transparente, avental emborrachado impermeável, luvas de nitrila, botas de borracha e máscara descartável com filtro de carvão ativado.

Foi considerada, para as botas e para as máscaras descartáveis com filtro de carvão ativado, a eficiência de controle das exposições de 95\%, conforme Lundehn et al. (1992).

Como medidas de proteção coletivas para o tratorista, avaliou-se a eficiência de uma semicabina acoplada à capota original do trator e duas cabinas totalmente fechadas e climatizadas.

A semicabina utilizada foi a fabricada pela Indústria Matão Equipamentos Industriais e Agricolas Ltda. Essa semicabina é fechada com estruturas de metal e vidro nas partes frontal e traseira, e parcialmente fechada nas laterais, na região do assento do trator. As aberturas laterais têm a função de propiciar a entrada e saída do operador no trator.

As duas cabinas fechadas avaliadas foram uma da marca Cabinas Real, de Novo Hamburgo-RS, e outra da marca Agro Leite - Irmãos Oliveira Leite LTDA, de lbirá-SP. Essas cabinas são totalmente fechadas com estruturas de metal e vidro, possuindo uma porta com fechadura em um dos lados do equipamento. Possuem um sistema climatizador de ar, posicionado sobre a capota, onde o ar é coletado, refrigerado e lançado no interior da cabina. Este sistema de refrigeração é dotado de filtros para impedir a entrada de partículas no interior da cabina. Os filtros utilizados nos climatizadores das cabinas não foram os específicos para agrotóxicos.

As exposições às caldas, quantificadas com os traçadores, foram utilizadas como dados substitutos, de acordo com Jensen (1984), para estimar as exposições aos agrotóxicos recomendados para aplicação nas condições avaliadas. Foram calculadas as EDPs e as ERPs do tratorista para as recomendações dos 21 agrotóxicos listados a seguir: acrinathrin, azocyclotin, benomil, bromopropylate, chlorothalonil, chlorpyrifós, cyhexatin, deltamethrine, dicofol, dimethoate, ethion, fenbutatin oxide, fenpyroximate, hexythiazox, methidathion, parathion methyl, propargite, thiophanate methyl, triazophos, triclorfon e vamidothion, considerando-se as respectivas maiores dosagens recomendadas (ANDREI, 1999). Para o preparador de caldas, foram calculadas as EDPs e as ERPs para as concentrações dos 18 produtos de formulação líquida listados a seguir: acrinathrin, bromopropylate, chlorothalonil, chlorpyrifós, cyhexatin, deltamethrine, dicofol, dimethoate, ethion, fenbutatin oxide, fenpyroximate, methidathion, parathion methyl, propargite, thiophanate methyl, triazophos, triclorfon e vamidothion, segundo Andrei (1999). Esses agrotóxicos foram escolhidos por serem os mais tóxicos ou os mais utilizados pelos citricultores nessas condições de pulverização.

A segurança das condições de trabalho com cada agrotóxico foi estimada por meio do cálculo da margem de segurança (MS) com a fórmula de Severn (1984) modificada por Machado Neto (1997), que é a seguinte: $M S=($ NOEL $\times 70) /(Q A E \times 10)$, onde: $M S=$ margem de segurança; $\mathrm{NOEL}=$ nivel de efeitos não observados $(\mathrm{mg} / \mathrm{kg} / \mathrm{dia})$ (TOMLIN, 1997); 70 = peso corpóreo médio $(\mathrm{kg}) ; 10=$ fator de segurança para compensar a extrapolação dos valores de NOEL obtidos em animais de laboratório para o homem (BROUWER et al., 1990); e QAE = quantidade absorvível das exposições avaliadas (mg/dia). No cálculo da MS na via dérmica, foi considerada a QAE $=10 \%$ da ED avaliada (0,1 x ED). No cálculo da MS na via respiratória, considerou-se $Q A E=$ $100 \%$ da ER avaliada. A MS total (ED + ER) foi calculada considerando a $Q A E=[(0,1 \times$ $E D)+(E R)]$. O critério utilizado para a classificação da segurança das condições de trabalho foi o seguinte: se MS 1, condição segura, exposição aceitável e risco tolerável; e se $M S<1$, condição insegura, exposição inaceitável e risco intolerável, segundo Machado Neto (1997).

\section{Resultados e discussão}

Os resultados das avaliações das exposições potenciais nas vias dérmica e respiratória às suspensões de cobre e de manganês, 
respectivamente, para o tratorista, sem e com medida de proteção, encontram-se na Tabela 1. Verifica-se que, na exposição total do tratorista, na fase l, a EDP foi responsável por $99,99 \%$ e a ERP por apenas $0,01 \%$ e, na fase II, a EDP correspondeu a $99,996 \%$ e a
ERP a 0,004\%. Esses resultados concordam com os de Wolfe et al. (1972) e de Van Hemmen (1992), onde estes autores relatam que $99 \%$, ou mais, da exposição total, ocorre na via dérmica e apenas $1 \%$, ou menos, na via respiratória.

Tabela 1 Exposições potenciais diárias, nas vias dérmica e respiratória, proporcionadas ao tratorista aplicando agrotóxicos na cultura de citros com um turbopulverizador, avaliadas em duas fases.

\begin{tabular}{lcc}
\hline \multirow{2}{*}{ Vias de Exposição } & \multicolumn{2}{c}{ Exposições em $\mathrm{mL}$ de calda por dia } \\
\cline { 2 - 3 } & \multicolumn{1}{c}{ fase I } & fase II \\
\hline Dérmica & 217,300 & 496,800 \\
Respiratória & 0,021 & 0,021 \\
\hline Total & 217,321 & 496,821 \\
\hline
\end{tabular}

A diferença entre as exposições avaliadas nas duas fases pode ser devida às diferentes condições ambientais durante os periodos de avaliação. Na fase l, a temperatura média foi de $29,7^{\circ} \mathrm{C}$ e a umidade relativa do ar média, de 73,3\%; na fase II, a temperatura média foi de $34,8^{\circ} \mathrm{C}$ e a umidade relativa do ar média, de $37,6 \%$. Portanto, este maior valor na fase ll não foi devido à temperatura e à umidade relativa do ar, pois, por esses parâmetros, esperava-se exposição menor nessa fase. Outros fatores podem ter influido na exposição na fase ll, como, por exemplo, a direção e velocidade do vento durante as avaliações das exposições e o porte das plantas.

A eficiência das medidas de proteção para - tratorista pode ser observada na Tabela 2 , onde se verifica que o conjunto AZR foi a medida de proteção mais eficiente, controlando $96,7 \%$ da exposição total. A semicabina controlou apenas $33,2 \%$ da exposição total. As cabinas totalmente fechadas, Real e Agroleite, controlaram 94,9 e $85,5 \%$, respectivamente, verificando-se que estes controles foram semelhantes ao proporcionado pelo conjunto $A Z R$, apenas com ligeira inferioridade para a cabina Agroleite.

Tabela 2 Exposições totais do tratorista aplicando agrotóxicos em citros com o turbopulverizador sem proteção e com a utilização das medidas de proteção, e respectivas eficiências de controle das exposiçōes, em porcentagem.

\begin{tabular}{cccc}
\hline & Equipamento de Proteção & $\begin{array}{c}\text { Exposição Total } \\
\text { (mL de calda/dla) }\end{array}$ & $\begin{array}{c}\text { Eficiência } \\
\text { (\%) }\end{array}$ \\
\hline$f$ & Sem Proteção & 217,32 & - \\
a & AZR & 7,20 & 96,7 \\
s & Semicabina & 145,11 & 33,2 \\
i & Sem Proteção & 496,82 & - \\
\hline$f$ & Cabina Real & 25,30 & 94,9 \\
a & S & 71,90 & 85,5 \\
o & Cabina Agroleite & II &
\end{tabular}


Na avaliação da fase I, verifica-se que $71,83 \%$ da EDP atingiu apenas três partes do corpo, em ordem decrescente: mãos, pés e coxas + pernas - atrás. Na fase II, verificase também que três partes do corpo foram as mais expostas: mãos, braços e cabeça + pescoço, em ordem decrescente, que receberam juntas $62,95 \%$ da EDP.

Considerando-se esses resultados, verificase que a segurança desse tratorista, através do uso de EPIs, deveria iniciar-se pela recomendação das vestimentas que protegem as mãos, pés e coxas + pernas - atrás, na fase l, e mãos, braços e cabeça + pescoço, na fase ll.

Tabela 3 Distribuição da exposição dérmica potencial nas partes do corpo do tratorista aplicando agrotóxicos em citros com o turbopulverizador e respectivas porcentagens em relação à exposição dérmica total avaliadas em duas fases.

\begin{tabular}{lcccccr}
\hline \multirow{2}{*}{ Partes do Corpo } & \multicolumn{2}{c}{ fase I } & & \multicolumn{2}{c}{ fase II } \\
\cline { 2 - 3 } \cline { 6 - 7 } & $\mathrm{mL}$ de calda por dia & \% & Total & & $\mathrm{mL}$ de calda por dia & \% Total \\
\hline Cabeça + pescoço & 2,81 & 1,29 & & 97,71 & 19,67 \\
Face & 18,41 & 8,47 & & 39,77 & 8,00 \\
Mãos & 79,24 & 36,46 & & 111,89 & 22,52 \\
Braços & 14,64 & 6,74 & & 103,14 & 20,76 \\
Tórax-frente & 10,46 & 4,81 & & 19,19 & 3,86 \\
Tórax-atrás & 4,07 & 1,87 & & 23,79 & 4,79 \\
Coxas+pernas-frente & 10,83 & 4,98 & & 13,30 & 2,68 \\
Coxas+pernas-atrás & 22,20 & 10,22 & & 18,32 & 3,69 \\
Pés & 54,67 & 25,15 & & 69,74 & 14,04 \\
\hline
\end{tabular}

Tabela 4 Exposições potenciais (EP) e não controladas (ENC) pelo conjunto AZR, nas vias dérmica e respiratória, em $\mathrm{mL} / 10$ tanques abastecidos, proporcionados pela atividade de abastecimento de um tanque de $2.000 \mathrm{~L}$ de capacidade, para o preparador de caldas, e respectiva eficiência de controle da exposição, em porcentagem, proporcionada pelo conjunto AZR.

\begin{tabular}{|c|c|c|c|}
\hline $\begin{array}{l}\text { Exposições em } \\
\mu \sqcup / 10 \text { tanques }\end{array}$ & $\begin{array}{c}\text { EP } \\
\text { Sem proteção }\end{array}$ & $\begin{array}{l}\text { ENC } \\
\text { AZR }\end{array}$ & $\begin{array}{c}\text { Eficiência } \\
(\%)\end{array}$ \\
\hline Dérmica & $1.798,2$ & 200,0 & \\
\hline Respiratória & 0,1 & 0,005 & \\
\hline Total & $1.798,3$ & 200,005 & 88,9 \\
\hline
\end{tabular}

Para o preparador de caldas, sem e com medida de proteção, os resultados das avaliações das EDs e das ERs às suspensões de cobre e de manganês, respectivamente, encontram-se na Tabela 4. Verifica-se que $99,994 \%$ da exposição total nessa atividade ocorre na via dérmica e apenas $0,006 \%$ na via respiratória. Esses resultados são semethantes aos avaliados para o tratorista realizando aplicações de agrotóxicos com o pulverizador turbopulverizador, e com os resul- tados citados na literatura especializada (WOLFE et al., 1972 e VAN HEMMEN, 1992).

A eficiência do Conjunto AZR no controle das exposições do preparador de caldas foi de $88,9 \%$. Esse resultado está de acordo com os niveis médios de controle das exposiçōes no trabalho proporcionadas pelas atividades com agrotóxicos citados por Lundehn et al. (1992).

Na Tabela 5, observa-se a distribuição da 
Tabela 5 Distribuição da EDP nas partes do corpo do preparador de caldas de um tanque de $2.000 \mathrm{~L}$ de capacidade $(\mathrm{mL} / 10$ tanques abastecidos) e respectivas porcentagens em relação à exposição total.

\begin{tabular}{lcc}
\hline \multicolumn{1}{c}{ Partes do Corpo } & $\mu \mathrm{L} \mathbf{1 0}$ tanques & \% Total \\
\hline Cabeça + pescoço & 14,40 & 0,80 \\
Face & 27,00 & 1,50 \\
Mãos & $1.544,40$ & 85,80 \\
Braços & 30,60 & 1,70 \\
Tórax-frente & 19,80 & 1,20 \\
Tórax-atrás & 12,60 & 0,70 \\
Coxas+pernas-frente & 34,20 & 1,90 \\
Coxas+pernas-atrás & 21,60 & 1,20 \\
Pés & 93,60 & 5,20 \\
\hline
\end{tabular}

EDP nas diversas partes do corpo do preparador de caldas abastecendo 10 tanques de $2.000 \mathrm{~L}$ de capacidade, e respectivos percentuais dessa exposição. Verifica-se que $85,8 \%$ da exposição dérmica total se concentrou nas mãos, que são as partes do corpo que entram em contato direto com as embalagens dos agrotóxicos.

Considerando-se esses resultados, a recomendação de medidas de proteção individual do preparador de caldas deve iniciar-se pelo uso de luvas impermeáveis, pois as partes que protegem, recebem $85,8 \%$ da exposição dérmica total.

Nos cálculos das MSs para o tratorista sem proteção, na fase l, verificou-se que foram seguras as recomendações dos 12 agrolóxicos seguintes: acrinathrin, benomil, bromopropylate, cyhexatin, deltamethrine, fenbutatin oxide, fenpyroximate, hexythiazox, propargite, thiophanate methyl, triclorfon e vamidothion. Com a utilização da semicabina, foram seguras 13 recomendações de agrotóxicos, além das citadas acima, mais a recomendação de chlorothalonil. Com o uso do conjunto AZR, foram seguras as recomendações de 18 agrotóxicos, além dos citados anteriormente, mais o azocyclotin, dicofol, dimethoate, ethion e methidathion.

Para o tratorista sem proteção, na fase II, foram seguras as recomendações de 8 agrotóxicos: acrinathrin, benomil, bromopropylate, fenbutatin oxide, fenpyroximate, hexythiazox, thiophanate methyl e vamidothion. Com a utilização da cabina Agroleite, foram seguras as recomendações de 14 agrotóxicos, além dos já citados, mais o azocyclotin, chlorothalonil, cyhexatin, deltamethrine, propargite e triclorfon. Com a cabina Real, foram seguras as recomendações de 16 agrotóxicos, além dos citados, mais o dicofol e ethion.

Para o preparador de caldas sem proteção, foram seguras as recomendações de 15 agrotóxicos: acrinathrin, bromopropylate, chlorothalonil, cyhexatin, deltamethrine, dicofol, dimethoate, ethion, fenbutatin oxide, fenpyroximate, methidathion, propargite, thiophanate methyl, triclorfon e vamidothion. Com a utilização do conjunto AZR, foram seguras as recomendações de 18 agrotóxicos, além dos citados anteriormente, mais o chlorpyrifós, parathion methyl e triazophos.

A eficiência das medidas de proteção foi evidenciada em relação à classificação das margens de segurança das recomendações de uso dos diversos agrotóxicos. Verifica-se que, devido à adoção de medidas de proteção, nas duas atividades estudadas, o número de recomendações seguras foi maior do que quando não se utilizaram medidas de proteção.

A composição da MS, para cada recomendação, sofreu influência da toxicidade intrínseca de cada um dos agrotóxicos e da exposição proporcionada pelas condições de trabalho aos trabalhadores (TURNBULL, 1985). 
O efeito da toxicidade do agrotóxico na segurança da condição de trabalho pode ser verificado dentro de uma mesma atividade realizada na aplicação de diferentes agrotóxicos, que resultam em diferentes valores de MS e, conseqüentemente, em diferentes classificações de segurança. $O$ efeito da exposição pode ser observado comparando-se a segurança na aplicação de um mesmo agrotóxico em atividades diferentes. A atividade pode ser segura para o tratorista $e$ insegura para o preparador de caldas; o fator determinante foi, nesse caso, a exposição sofrida por esses trabalhadores, mais precisamente a exposição proporcionada pela condição específica de trabalho de cada operador.

\section{Conclusões}

Considerando-se os resultados obtidos nesse trabalho, pode-se concluir que, para o tratorista pulverizando agrotóxicos em citros com o turbopulverizador, a exposição potencial total (dérmica + respiratória) foi de 217,3 e de 496,8 $\mathrm{mL}$ de calda por dia nas fases l e II, respectivamente, sendo as mãos a região mais exposta do corpo nas duas fases; para o preparador de caldas, a exposição potencial total foi de $1.798,3 \mathrm{~mL}$ do produto formulado por 10 tanques preparados, sendo, também, as mãos a região mais exposta do corpo desse trabalhador. As medidas de proteção mais eficientes, para o tratorista, foram o conjunto AZR e a cabina Real e, para o preparador de caldas, o conjunto AZR foi eficiente no controle da exposição, pois foi suficiente para tornar as condições de trabatho inseguras em seguras. Classificaram-se como seguras para tratorista, na fase $l$, sem proteção (EDP), 12 recomendações, $13 \mathrm{com}$ a utilização da semicabina e, com o conjunto AZR, 18 recomendações; e na fase Il, sem proteção (EDP), 8 recomendações, $14 \mathrm{com}$ o uso da cabina Agroleite e, com a cabina Real, 16 recomendações, das 21 recomendações de agrotóxicos estudadas. Para o preparador de caldas sem proteção (EDP), foram seguras 15 recomendações e, $18 \mathrm{com}$ a utilização do conjunto AZR, das 18 recomendaçōes de agrotóxicos estudadas.

\section{Referências bibliográficas}

\section{ANDREI, E. (ed.). Compêndio de defen- sivos agrícolas: guia prático de pro- dutos fitossanitários para uso agrícola. São Paulo: Organização Andrei Editora LTDA, 1999. 672p.}

BROUWER D.H. et al. Respiratory exposure to field-strength dusts in greenhouses during application and after re-entry. Annual report 1990, TNO Health Research, p. 183-184, 1990.

\section{BULL, D.; HATHAWAY, D. Pragas e ve-} nenos: agrotóxicos no Brasil e no terceiro mundo. Petrópolis: Vozes, 1986. $235 p$.

JENSEN, J.K. The assumptions used for exposure assessments. In: SIEWIERSKI, $M$. (ed.). Determination and assessment of pesticide exposure. New York: Elsevier, 1984. p. 147-152.
LUNDEHN, J. et al. Uniform principles for safeguarding the health of applicators of plant protection products (Uniform principles for operator protection). Berlin: Kommissionsverlag Paul Parey, 1992. 90p.

MACHADO NETO, J.G. Estimativas do tempo de trabalho seguro e da necessidade de controle da exposição dos aplicadores de agrotóxicos. 1997. 83f. Tese (LivreDocência) - Faculdade de Ciências Agrárias e Veterinárias, Universidade Estadual Paulista, Jaboticabal, 1997.

MACHADO NETO, J.G.; MATUO, T. Avaliação de um amostrador para o estudo da exposição dérmica potencial de aplicadores de defensivos agrícolas. Ciência Agronômica, Jaboticabal v. 4, n. 2, p. 21-22, 1989. 
MATUO, T. Desenvolvimento de um pulverizador intermitente operado fotoeletricamente para tratamento de pomares de citros. 1988. 167f. Tese (Livre-Docência) - Faculdade de Ciências Agrárias e Veterinárias, Universidade Estadual Paulista, Jaboticabal, 1988.

OLIVEIRA, M.L. Segurança no trabalho de aplicação de agrotóxicos com turboatomizador e pulverizador de pistolas em citros. 2000. $99 f$. Dissertação (Mestrado) - Faculdade de Ciências Agrárias e Veterinárias, Universidade Estadual Paulista, Jaboticabal, 2000 .

PRADELA, V. A. Segurança dos aplicadores de glifosate em jato dirigido em cultura adulta de cana-de-açúcar com pulverizadores costais. 1998. 36f. Monografia (Monografia em Engenharia de Segurança do Trabalho) Centro Universitário Moura Lacerda, Câmpus de Ribeirão Preto, Ribeirão Preto, 1998.

SEVERN, D.J. Use of exposure data for risk assessment. In: Siewierski M (ed.). Determination and assessment of pesticide exposure. New York: Elsevier, 1984. p. 13-19.

SINDAG - Sindicato da Indústria de Produtos para Defesa Agrícola. Vendas de defensivos agrícolas por cultura de destinação e classes. São Paulo. Disponivel em: <http:// www.sindag.com.br/db/arqs/CULTURAS-9700.xls $\geq$. Acesso em: marco de 2002.

TOMLIN, C.D.S. (ed.). The pesticide manual: a world compendium. 11.ed. London: BCPC, 1997. 1.606p.

TURNBULL, G.L. Current trends and future needs. In: TURNBULL, G.L. (ed.). Occupational hazards of pesticide use. London: Taylor \& Francis, 1985. p. 99-116.

VAN HEMMEN, J.J. Agricultural pesticide exposure data bases for risk assessment. Rev Environ Contam Toxicol, New York, v. 126, p. 1-85, 1992.

WOLFE, H.R. et al. Exposure of spraymen to pesticide. Arch Environ Hith, v. 25, p. 29-31, 1972. 